ABSTRACTED SCIENTIFIC CONTENT

For personal use only. Not to be reproduced without permission of the publisher (editorial@gabi-journal.net).

\section{Weighing up the cost of switching to biosimilars}

It is far from clear whether off-patent biological medicines can offer the same cost savings as those offered by off-patent nonbiological (chemically derived) medicines, write a group of Brussels-based health economists in the European Journal of Health Economics. Several key factors will play a role in realizing the potential of these effective low-cost agents, including regulatory issues, price and reimbursement policies and, ultimately, whether physicians can be assured of their safety and effectiveness [1].

Biosimilar sales represent a relatively small proportion of the EU pharmaceutical market, but this is increasing steadily. The price difference between biological originator medicines and their biosimilars currently stands at between 10 and 35\%, which varies according to country and medicine. Price differences of up to $80 \%$ have been attributed to generic competition, but theoretical models predict that biosimilar competition will lead to less dramatic price reductions.

Most data on biosimilar uptake is provided by the US-based healthcare information firm IMS data. A recent study used IMS data to provide estimates on biosimilar-related savings between 2007 and 2020 for eight European countries (France, Germany, Italy, Poland, Romania, Spain, Sweden, and UK) [2]. Predicted savings related to biosimilar monoclonal antibodies ranged between Euros 11.8 billion and Euros 33.4 billion. But these estimates relied heavily on assumptions about the German pharmaceutical market, write Maria-Isabel Farfan-Portet et al. [1], and on large predicted savings with monoclonal antibodies - for which biosimilars are not yet available.

Clearly, considerable savings could be made with the introduction of biosimilars, but there are several obstacles to their uptake. Regulatory issues, biosimilar acceptability among physicians, price and reimbursement policies as well as supply and demand-side incentives will finally determine the level of biosimilar-related savings.

Effective measures need to be put in place in order to improve biosimilar uptake, write Farfan-Portet et al. One issue that needs addressing is that making prescriptions using the International Nonproprietary Name (INN), or generic name, is not currently an option for biosimilar medicines. They are biosimilars not 'bioidenticals'. INN prescribing has driven the ever increasing uptake of generic medicines, and now policymakers must work towards putting a suitable alternative system in place for biosimilars.

Physicians may also be wary of switching patients to biosimilars if those patients have chronic disease and have been long-term users of an originator drug. Even when patients have not been on the originator drug previously, physicians may not be given sufficient information and may overlook the financial advantages of prescribing biosimilars.

The two markets - generics and biosimilars - behave differently. Ruling out this segmentation, to the point where the price reductions seen with generics are mirrored by biosimilars, will depend on experience with biosimilars. It is important that healthcare workers are supplied with clinical data that proves the effectiveness and safety of biosimilars, particularly where this involves switching. At the same time, prescribers must be made aware of all the advantages of these lower-cost medicines.

Such views echo those expressed elsewhere, for example, by Ebbers et al. writing in the journal Drug Discovery Today [3]. Writing in 2012, Ebbers et al. noted that concerns about the safety and efficacy of biosimilars had dissuaded many healthcare professionals from prescribing them. He and his co-authors suggested increased engagement between regulatory authorities and the medical community over the drafting of regulatory guidelines.

In the case of Europe, Ebbers et al. suggested that a platform should be established to facilitate dialogue with European medical associations on issues of common interest, which could promote the acceptance of regulation. Failing to involve doctors when putting together regulations would slow down the acceptance of 'the cost-effective and innovative medicinal products of the future', Ebbers says [4].

With the expiry of market exclusivity of major biological blockbusters, many of the companies that produce those blockbusters are starting to develop their own biosimilars. This could cause significant changes to the pattern currently seen. These companies are already significantly experienced in the production of complex biologicals. Their experience could well lead to increasingly optimized production of biosimilars at low cost. This could even lead to further optimized production of originator molecules, all of which could only increase the likelihood of price cuts for biosimilars.

It is likely that originator companies will also produce biosimilar monoclonal antibodies, and are likely to have different marketing strategies than those employed by the companies that currently make biosimilars. It remains an open question, writes Farfan-Portet, whether these companies will use the same strategies and provide similar levels of information services for their innovator products and for biosimilars. The addition of originator companies to the biosimilar story could well change the current perception of biosimilars and, they write, even change the current biosimilar business model.

'Lack of market penetration of the currently available biosimilars may be seen as a lost opportunity, less in terms of current savings than as a barrier for potential future savings,' writes FarfanPortet. The question of how much money will be saved will rely on regulatory issues, whether physicians will accept biosimilars, and price and reimbursement policies. 'The challenge for policy makers in the coming years will be to set effective measures leading to improved biosimilar uptake,' they conclude.

\section{Competing interests: None.}

Provenance and peer review: Article abstracted based on published scientific or research papers recommended by members of the Editorial Board; internally peer reviewed.

Bea Perks, PhD, GaBI Journal Editor

\section{References}

1. Farfan-Portet MI, Gerkens S, Lepage-Nefkens I, Vinck I, Hulstaert F. Are biosimilars the next tool to guarantee cost-containment for pharmaceutical expenditures? Eur J Health Econ. 2014;15(3):223-8.

References $2-4$ can be found on page 87 . 


\section{Weighing up the cost of switching to biosimilars}

References (please see the full manuscript on page 100)

2. Haustein R, de Millas C, Höer A, Häussler H. Saving money in the European healthcare systems with biosimilars. Saving money in the European healthcare systems with biosimilars. Generics and Biosimilars Initiative Journal
(GaBI Journal). 2012;1(3-4).120-6. doi: 10.5639/ gabij.2012.0103-4.036

3. Ebbers HC, Pieters T, Leufkens HG, Schellekens H. Effective pharmaceutical regulation needs alignment with doctors. Drug Discov Today. 2012;17(3-4):100-3.
4. The potential for doctors to contribute to biosimilar guidelines. Generics and Biosimilars Initiative Journal (GaBI Journal). 2012;1(3-4):152. doi: 10.5639/gabij.2012.0103-4.039 DOI: $10.5639 /$ gabij.2014.0302.025 Copyright (c) 2014 Pro Pharma Communications International 